\title{
Upper and lower esophageal sphincter kinetics are modified during maturation: effect of pharyngeal stimulus in premature infants
}

\author{
Sudarshan R. Jadcherla ${ }^{1,2}$, Theresa R. Shubert², Ish K. Gulati, ${ }^{1,2}$, Preceousa S. Jensen ${ }^{1,2}$, Lai Wei ${ }^{3}$ and Reza Shaker ${ }^{4}$
}

BACKGROUND: We hypothesized that changes in proximal and distal esophageal sphincter kinetics evoked upon pharyngeal provocation undergo longitudinal maturation.

METHODS: Pharyngeal stimulation-induced reflexes were characterized using novel pharyngo-esophageal motility methods in 19 healthy premature neonates, studied at $34.7 \pm 0.8 \mathrm{wk}$ (time-1) and $39.3 \pm 1.1 \mathrm{wk}$ postmenstrual age (time-2). Graded volumes of air (290 infusions) and sterile water (172 infusions) were infused to define sensory-motor characteristics of upstream (pharyngeal reflexive swallow, PRS) and downstream (pharyngo-lower esophageal sphincter relaxation reflex, PLESRR) esophageal reflexes. Data displayed as mean \pm SE.

RESULTS: Threshold volumes were similar with air and water for PRS and PLESRR at time-1 and time-2. Multiple PRS responses were noted with water stimulus and were different between the media (time-1 vs. air, $P<0.0001$; time- 2 vs. air, $P=0.0003)$. Dose-response relationships for water were significant $(P<0.01$ for PRS and PLESRR time-1 and time-2), but not with air.

CONCLUSION: Significantly, the recruitment frequency of PRS and PLESRR increases with maturation, liquid is a superior medium for evoking such swallowing reflexes, and stimulusresponse relationships for these reflexes are evident. These changes in aerodigestive protective reflexive activity may indicate differences in modulation of excitatory and inhibitory pathways during longitudinal postnatal maturation.

O ropharyngeal dysphagia is a common and complex manifestation of many neonatal primary diagnoses; therefore, determining the prevalence can be challenging. Studies suggest it affects up to $60 \%$ of the NICU population $(1,2)$. Chronic dysphagia in premature infants leads to long term tube feeding strategies, thus impacting overall growth and development, in addition to escalating parental stress and healthcare costs $(3,4)$. Interventions to improve the oral feeding performance are necessary; however, the role of stimulation induced upper and lower esophageal sphincter and esophageal reflexes during maturation is unknown. Such information is vital for the assessment of safe feeding paradigms.

Proper function during the pharyngeal phase of swallowing is critical. Fetal ultrasounds demonstrate the presence of pharyngeal phase swallowing activity by $\sim 11 \mathrm{wk}$ gestation; however, the pharynx continues to develop structurally and functionally (5). Given the rise in premature births, understanding the adaptive pharyngeal function relative to bolus transit and airway protection is crucial in developing therapies that are more effective. Pharyngo-upper esophageal sphincter interactions in healthy preterm infants have been characterized by us as well as others $(6,7)$. Specifically, pharyngeal reflexive swallowing (PRS) reflexes occurred more frequently than pharyngo-upper esophageal sphincter contractile response reflexes, with a reflex response rate greater for water than for air in response to infusions to the pharynx (7). Characterization of the sensory-motor properties of the reflexes implicates the existence of afferent and efferent neural pathways resulting in these swallowing and airway protective functions. However, longitudinal changes in the development of these PRS reflexes are unknown. Furthermore, the effects of pharyngeal provocation on lower esophageal sphincter (LES) kinetics during maturation are also unknown.

The rationale for the current study is to investigate the role of pharyngo-esophageal sensory-motor interactions during maturation in the premature infant, and to augment the understanding of adaptive aerodigestive reflexes modulated by cranial nerves IX and X. Therefore, our aims were to test the hypothesis that pharyngeal stimulation induced upper esophageal sphincter (UES) and LES kinetics are dependent on the physical properties of the stimulus (air, water, and volume) and maturation. Specifically, we characterized the maturational changes in neonatal sensory-motor characteristics by defining the threshold volume, frequency recruitment, response latency, response magnitude, and response type. At time-1,

\footnotetext{
'Department of Pediatrics, Sections of Neonatology, Pediatric Gastroenterology and Nutrition, The Ohio State University College of Medicine, The Research Institute at Nationwide Children's Hospital, Columbus, Ohio; ${ }^{2}$ The Neonatal and Infant Feeding Disorders Program, The Research Institute at Nationwide Children's Hospital, Columbus, Ohio; ${ }^{3}$ Center for Biostatistics, The Ohio State University College of Medicine, Columbus, Ohio; ${ }^{4}$ Division of Gastroenterology and Hepatology, Medical College of Wisconsin, Milwaukee, Wisconsin. Correspondence: Sudarshan R. Jadcherla (sudarshan.jadcherla@nationwidechildrens.org) 
all infants had a gavage tube and were gavage fed exclusively, whereas at time-2, the majority of these infants had oral feeding skills. The rationale for the targeted use of graded volumes of air and water stimuli was to simulate the composition of a swallowed bolus in a safe experimental setting.

\section{RESULTS}

\section{Participant Characteristics}

Thirty-eight studies were performed in 19 enterally fed preterm infants $(29.1 \pm 1.1 \mathrm{wk}$ gestational age) longitudinally, at $34.7 \pm 0.8 \mathrm{wk}$ (IQR: $32.3-35.6 \mathrm{wk}$ ) and again at $39.3 \pm 1.1 \mathrm{wk}$ (IQR: 36.2-40.2 wk) postmenstrual age (PMA). All infants were healthy at either study, and were PMA appropriate for growth. At time-1 and time-2 respectively, weights were $1.8 \pm 0.2 \mathrm{~kg}$ and $2.6 \pm 0.2 \mathrm{~kg}(P<0.001)$, lengths were $42 \pm 1.1$ and $46 \pm 1.0 \mathrm{~cm}(P<0.001)$, and head circumferences were $29.8 \pm 0.7$ and $33.6 \pm 0.6 \mathrm{~cm}(P<0.001)$. All subjects were evaluated by the principal investigator (SRJ) and the attending neonatologist and were deemed healthy at study. None of the subjects had a presumed or proven clinical diagnosis of GER, and none were receiving prokinetics, acid suppressive therapy or xanthines at the time of study or discharge.

\section{Pharyngeal Reflexive Swallow}

Examples of stimulus induced PRS during maturation are shown in Figure 1a-d. The sensory-motor characteristics of PRS are shown in Figure 2. Specifically, response latency to PRS (Figure 2a), frequency of PRS (Figure 2b), frequency of complete propagation (Figure 2c), and frequency of multiple PRS (Figure 2d), media and maturational relationships are defined.

Sensory thresholds. The threshold volumes (mean \pm SD) for air at time- 1 and time- 2 respectively were $0.4 \pm 0.6$ vs. $0.2 \pm 0.2 \mathrm{ml}$. For water, threshold volumes at time- 1 and time- 2 respectively were $0.2 \pm 0.1$ vs. $0.1 \pm 0.1 \mathrm{ml}$. Comparisons within and between the maturational groups, as well as within and between the media were all not significant.

Sensory-motor characteristics during maturation. Air stimuli recruited PRS more quickly (vs. water) during both time-1 $(2.2 \pm 0.3$ vs. $3.0 \pm 0.3 \mathrm{~s})$ and time- $2(1.7 \pm 0.2$ vs. $3.0 \pm 0.2 \mathrm{~s})$; although there were no maturational differences within the media (Figure 2a). Water stimuli recruited PRS more frequently (vs. air) during both time-1 and time-2 (Figure 2b), however maturational comparisons were not significant ( $P=0.3$ and $P=0.2$ for air and water respectively for maturational comparisons). Air stimuli resulted in more completed PRS events (vs. water) at both time-1 and time-2; however, $P=$ NS for maturational comparison (Figure 2c). Multiple PRS occurred more often with water infusion (vs. air) at both time- 1 and time-2; however, $P=1.0$ for air time- 1 vs. time- 2 and $P=0.7$ for water time-1 vs. time-2 (Figure $2 \mathrm{~d}$ ).

Stimulus-volume reflex response relationships during maturation. The relationships involving the ability of stimulus media (air and water) and stimulus strength (volumes) across maturation are shown in the table (Table 1). Briefly, volumeresponse relationships were evident with water only for PRS at time-1 $(P<0.0001)$ and time-2 $(P=0.002)$.

\section{Pharyngo-Lower Esophageal Sphincter Relaxation Reflex}

Examples of stimulus induced PLESRR during maturation are shown in Figure 1a-d. The sensory-motor characteristics of PLESRR are shown in Figure 2. Specifically, response latency to PLESRR (Figure 2e), frequency of PLESRR (Figure 2f), duration of LES relaxation nadir (Figure 2g), and magnitude of LES relaxation (Figure $2 \mathbf{h}$ ), media and maturational relationships are defined.

Sensory thresholds. The threshold volumes (mean \pm SD) for air at time- 1 and time- 2 respectively were $0.3 \pm 0.3$ and $0.2 \pm 0.2 \mathrm{ml}$. For water, threshold volumes at time- 1 and time- 2 respectively were $0.1 \pm 0.1$ vs. $0.2 \pm 0.1 \mathrm{ml}$. Comparisons within and between the maturational groups, as well as within and between the media were all not significant.

Sensory-motor characteristics during maturation. Air stimuli recruited PLESRR more quickly (vs. water) at time-2 (3.3 \pm 0.4 vs. $5.1 \pm 0.5 \mathrm{~s}$ ), although there were no maturational differences in response latency within the media (Figure $2 \mathrm{e}$ ). In addition, water stimuli increased the recruitment frequency of PLESRR with maturation (Figure 2f). For PLESRR recruitment frequency between the media, $P=0.3$ at time- 1 and $P=0.07$ at time-2. PLESRR nadir duration was greater with water (vs. air) during time-1 $(13.1 \pm 1.2$ vs. $6.4 \pm 1.1 \mathrm{~s})$, as well as during time-2 (8.2 \pm 1.1 vs. $4.0 \pm 1.0 \mathrm{~s}$, Figure $2 \mathrm{~g})$. PLESRR nadir duration with water decreased with maturation (13.1 $\pm 1.2 \mathrm{~s}$ time- 1 vs. $8.2 \pm 1.1 \mathrm{~s}$ time-2, Figure $2 \mathrm{~g}$ ). Water stimuli resulted in increased magnitude PLESRR (vs. air) during time-2 (Figure 2h). PLESRR magnitude was also greater with water time-1 vs. water time-2 (Figure $2 \mathrm{~h}$ ). For PLESRR nadir pressure, $P=0.1$ for air time- 1 vs. time- 2 and $P=0.1$ for water time- 1 vs. time2. For air vs. water at time-1, $P=0.2$ and for air vs. water at time-2, $P=0.1$.

Stimulus-volume reflex response relationships during maturation. The relationships involving the ability of stimulus media (air and water) and stimulus strength (volumes) across maturation are shown in the table (Table 2). Briefly, volume-response relationships were evident with water only for PLESRR at time-1 $(P=0.004)$ and time-2 $(P<0.0001)$. Examples of volume-response characteristics with water are shown in Figure 3.

\section{DISCUSSION}

As is demonstrated by the current results, sensory-motor properties of pharyngeal reflexive swallowing (PRS) are similar between the groups longitudinally, while pharyngo-LES relaxation reflex (PLESRR) properties accelerate and become robust with maturation in preterm infants. Neither maturational stage, nor infusion media caused variation in threshold volume necessary to elicit these reflexes. At both times 
a

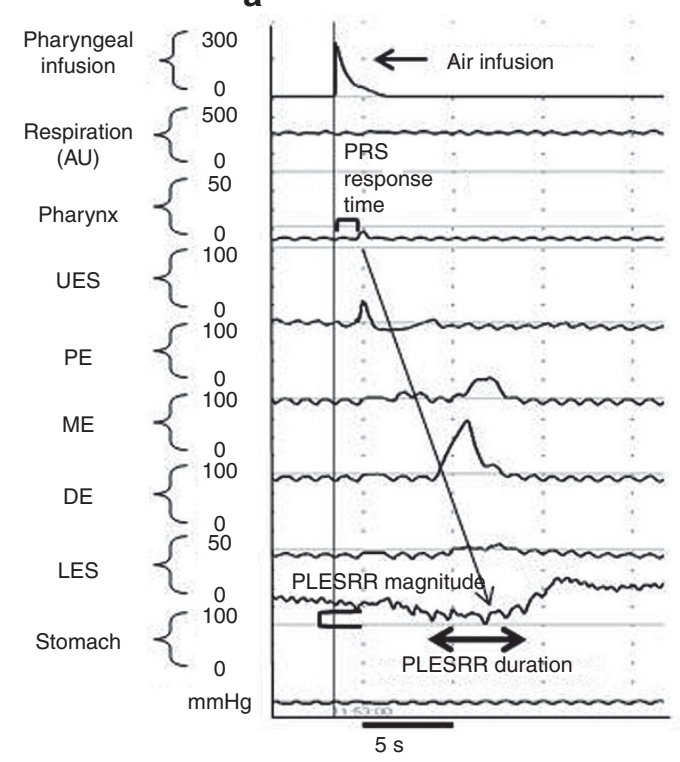

C

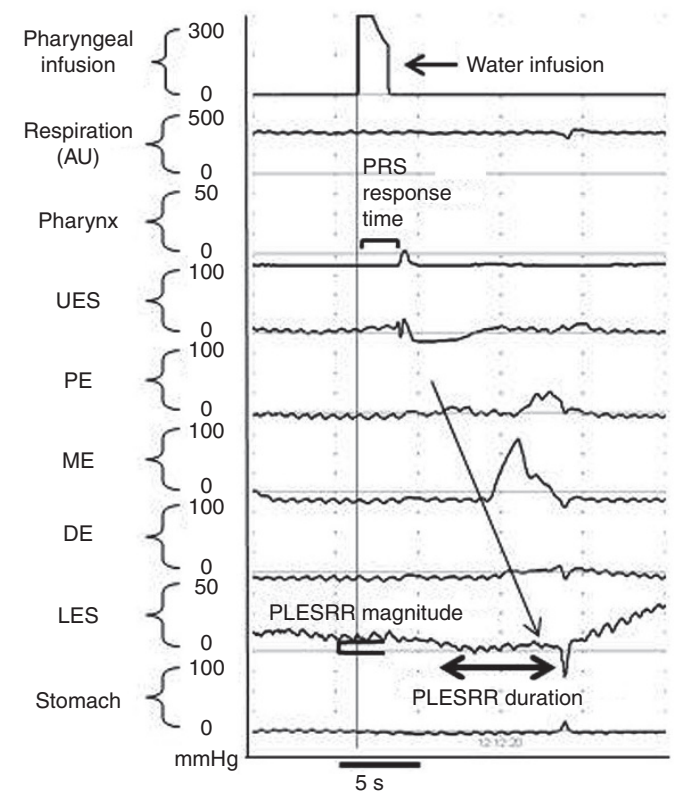

b

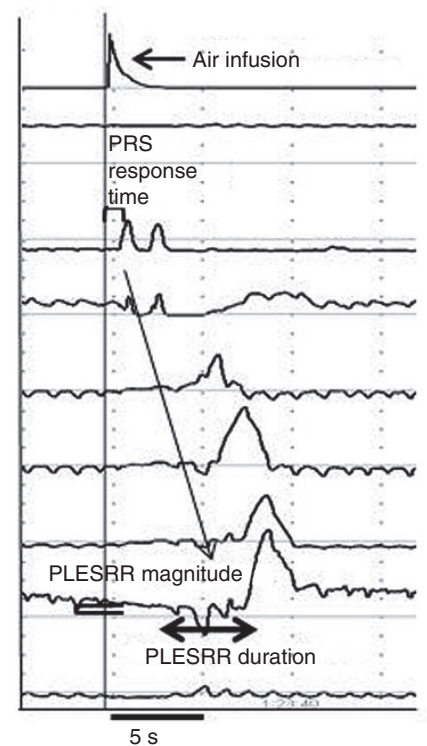

d

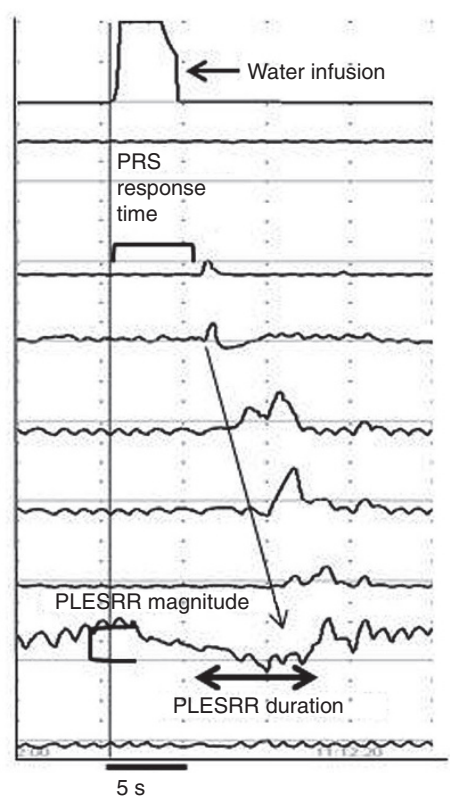

Figure 1 Maturational comparison of responses to air and water pharyngeal infusion. (a) Response to air infusion 35 wk postmenstrual age. (b) Response to air infusion $39 \mathrm{wk}$ postmenstrual age. (c) Response to water infusion $35 \mathrm{wk}$ postmenstrual age. (d) Response to water infusion $39 \mathrm{wk}$ postmenstrual age. Maturational differences are evident in both air and water infusion responses. Notice more rapid PRS onset in response to air infusions vs. water during both time-1 and time-2. Additionally, PLESRR onset time significantly decreases in response to air with time-2 infusions (vs. time-1). The magnitude of PLESRR is greater in response to water (vs. air) during time-2, as well as compared to water time-1. The duration of PLESRR is increases in response to water infusions (vs. air) at each time; however, PLESRR duration in response to water infusions decreases with maturation. LES, lower esophageal sphincter; PLESRR, pharyngo-lower esophageal sphincter relaxation reflex; PRS, pharyngeal reflexive swallow; UES, upper esophageal sphincter.

of study, infants demonstrated decreased response latency to PRS with air infusions (vs. liquid). For PLESRR, decreased response latency to air (vs. liquid) only occurred after maturation. Liquid was able to evoke PRS with greater frequency during both times of study. There was a maturational increase in liquid recruitment of PLESRR, but no difference vs. air at either time. For PLESRR, there was increased response magnitude for liquids at time-2 only (vs. air). This observation of increased PLESRR magnitude was also noted for time-1 liquid vs. time-2 liquid. Duration of PLESRR was increased for liquids (vs. air) at time-1 and time-2. PLESRR duration decreased for liquids upon comparison of time- 1 vs. time-2. Air is more effective at eliciting a greater rate of completely propagated solitary swallows (vs. liquid). Liquid is more effective for eliciting multiple PRS responses (vs. air). These findings are suggestive of skeletal muscle afferent osmo- vs. mechano-receptor differential ability present at a younger age; however this ability for smooth muscle receptors develops later.

Strong aerodigestive clearance mechanisms are essential for the development of safe neonatal feeding skills. Specifically, the 
a

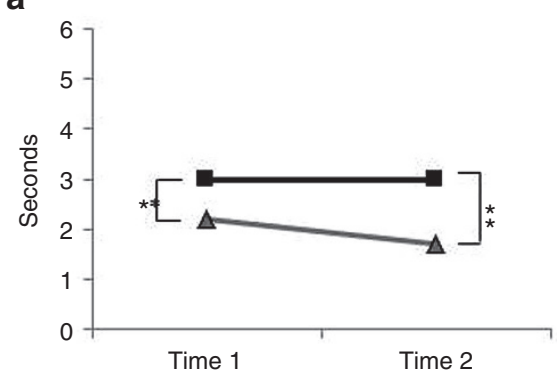

C

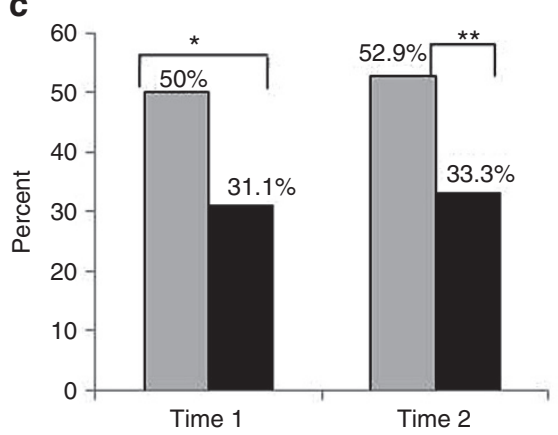

e
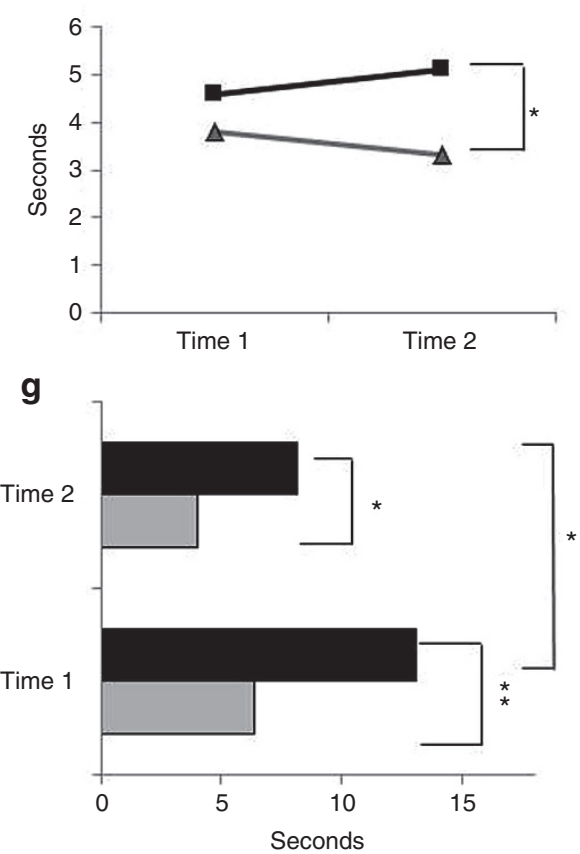

b

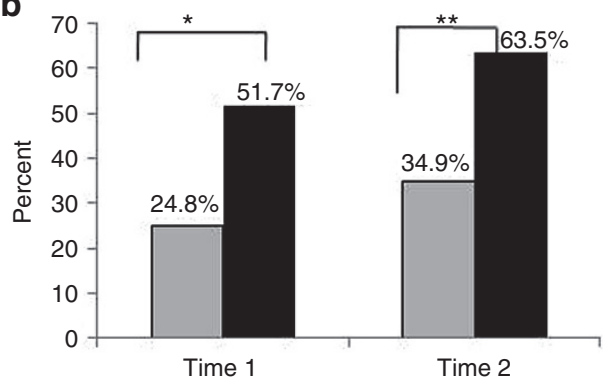

d

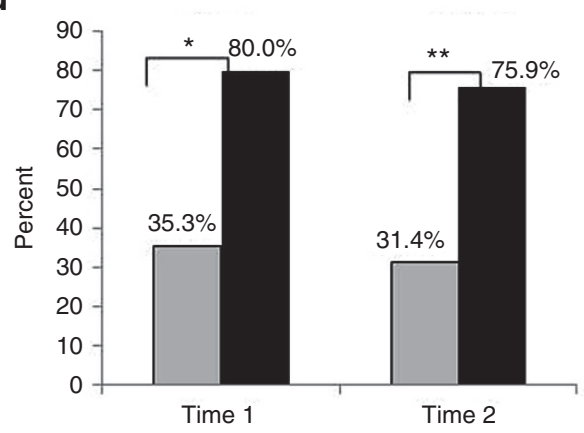

f

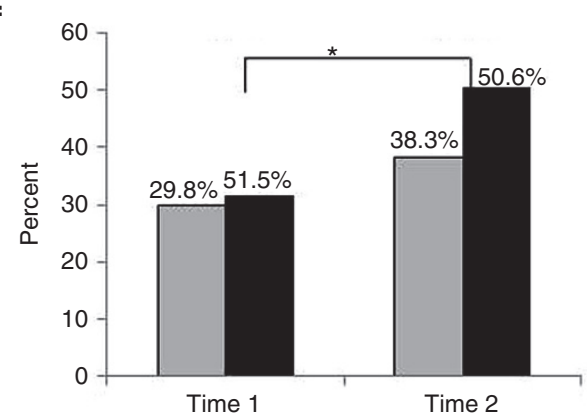

h

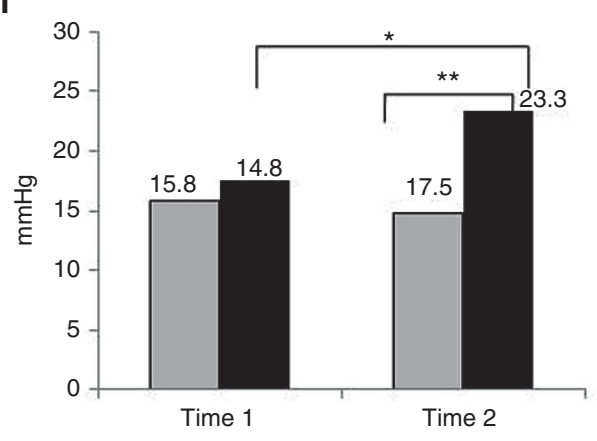

Figure 2 Characterization of media and maturational responses. (a) Response latency to pharyngeal reflexive swallow (in seconds); ${ }^{*} P=0.04 ; * * P=0.007$; triangle with a stroke: air; square with a stroke: water. (b) Frequency of pharyngeal reflexive swallow, $\%$; ${ }^{*} P=0.0004$; ${ }^{* *} P=0.002$; gray box: air; black box: water. (c) Frequency of complete propagation, $\%$; ${ }^{*} P=0.01 ; * * P=0.007$; gray box: air; black box: water. (d) Frequency of multiple pharyngeal reflexive swallows; \%; ${ }^{*} P<0.0001 ;{ }^{* *} P=0.0003$; gray box: air; black box: water. (e) Response latency to pharyngo-LES relaxation reflex (in seconds); ${ }^{*} P=0.05$; triangle with a stroke: air; square with a stroke: water. (f) Frequency of pharyngo-LES relaxation reflex, $\%$; ${ }^{*} P=0.01$; gray box: air; black box: water. (g) Duration of LES nadir (in seconds); ${ }^{*} P=0.05 ;{ }^{*} P=0.006$; gray box: air; black box: water. (h) Magnitude of LES relaxation; $\mathrm{mmHg} ;{ }^{*} P=0.03$; ${ }^{* *} P=0.05$; gray box: air; black box: water. Infants respond differently to air vs. water stimulus with maturation (time 1-35 wk postmenstrual age vs. time 2-39 wk postmenstrual age). Panels a-d characterize PRS responses to both media and maturation. Panels $\mathbf{e}-\mathbf{h}$ characterize PLESRR responses to both media and maturation. PLESRR, pharyngo-lower esophageal sphincter relaxation reflex; PRS, pharyngeal reflexive swallow.

PRS and the PLESRR aid in clearing esophageal content, and together with esophageal peristalsis, enhance the rapid anterograde transit into the stomach, thus minimizing concern for aspiration. Other neonatal proximal aerodigestive protective reflexes have been previously characterized with regard to maturation, indicating that in response to esophageal provocation (8-11), maturational changes are evident with postnatal development of preterm infants. The results from the current study 
Table 1 Stimulus-volume pharyngeal reflexive swallow recruitment across maturation

\begin{tabular}{llcccc}
\hline \% PRS response & Medium & $0.1 \mathrm{ml}$ & $0.3 \mathrm{ml}$ & $0.5 \mathrm{ml}$ & $\begin{array}{c}P \text { value for } \\
\text { volume effect }\end{array}$ \\
\hline Time-1 & Air & 22.6 & 34.8 & 22.2 & 0.9 \\
& Water* & 25.0 & 66.7 & 100.0 & $<0.0001$ \\
Time-2 & Air & 32.4 & 33.3 & 40.6 & 0.5 \\
& Water** & 44.4 & 72.7 & 93.3 & 0.002 \\
\hline
\end{tabular}

PRS, pharyngeal reflexive swallow.

${ }^{*} P<0.0001$ vs. air, ${ }^{* *} P=0.02$ vs. air. Water stimuli evoke more PRS responses (vs. air) at time-1 and time-2. Additionally, water evokes more PRS responses with increasing volumes.
Table 2 Stimulus-volume lower esophageal sphincter relaxation response recruitment across maturation

\begin{tabular}{llcccc}
\hline \% PLESRR & Medium & $0.1 \mathrm{ml}$ & $0.3 \mathrm{ml}$ & $0.5 \mathrm{ml}$ & $\begin{array}{c}\text { Pvalue for } \\
\text { volume effect }\end{array}$ \\
\hline Time-1 & Air & 19.4 & 43.5 & 30.6 & 0.2 \\
& Water & 19.1 & 43.3 & 46.2 & 0.004 \\
Time-2 & Air & 32.4 & 52.4 & 40.6 & 0.5 \\
& Water* & 33.3 & 56.3 & 85.7 & $<0.0001$ \\
\hline
\end{tabular}

PLESRR, pharyngo-lower esophageal sphincter relaxation reflex.

${ }^{*} P=0.008$ vs. air. Water stimuli evoke PLESRR more often as infusion volume increases during both time-1 and time-2. Additionally, water evoked more PLESRR (vs. air) during time-2.
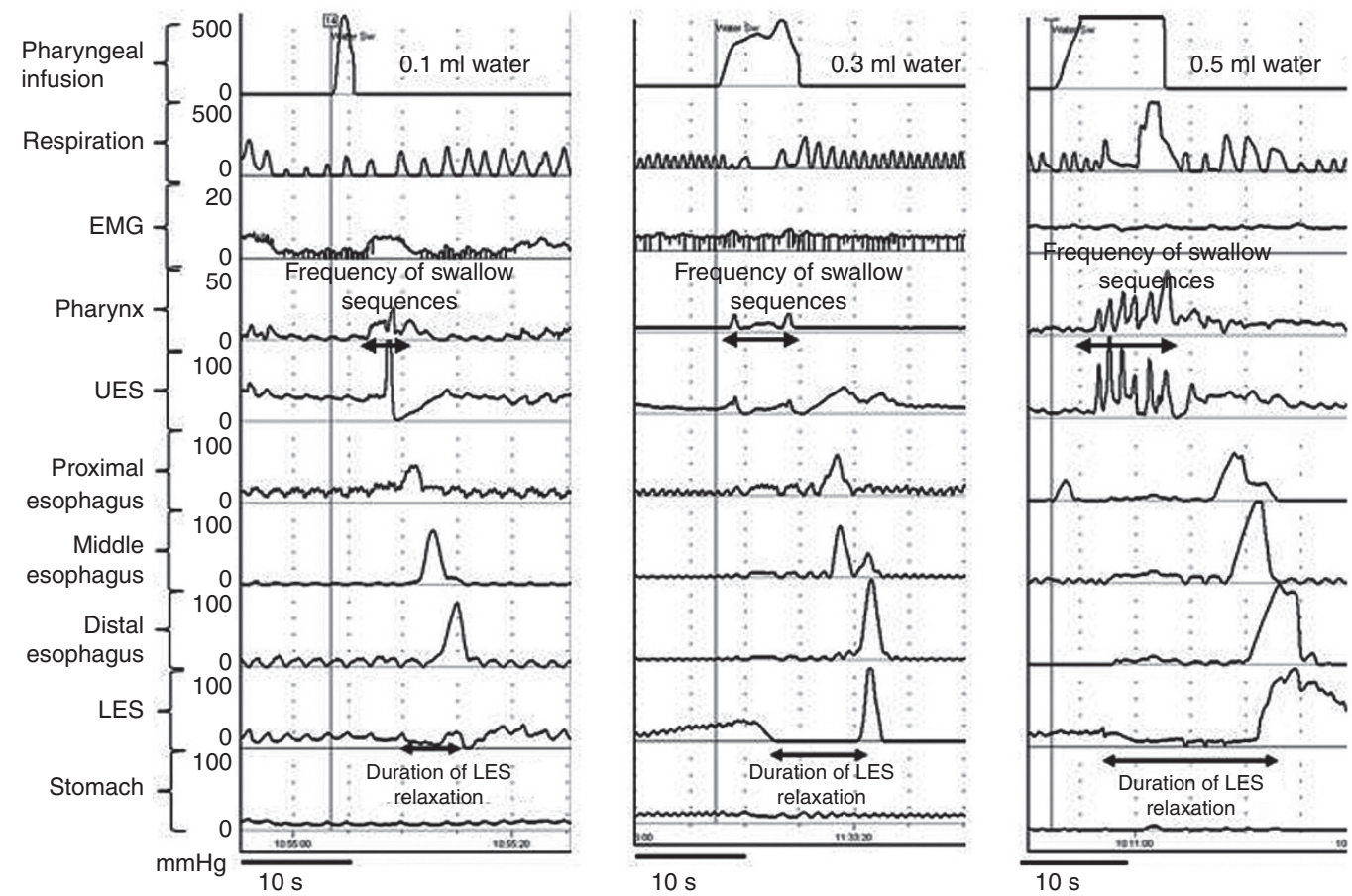

Figure 3 Volume-dependent pharyngeal reflexive swallow and PLESRR frequency. Note the graded infusion volumes given to the pharynx, presence of multiple pharyngeal peaks with increasing volume, and increasing duration of PLESRR with increasing infusion volume. LES, lower esophageal sphincter; PLESRR, pharyngo-lower esophageal sphincter relaxation reflex; UES, upper esophageal sphincter.

show a lack of maturational differences in the characteristics of PRS. This could be due to (i) Similar maturation of skeletal muscle dependent proximal reflexes at the studied time periods in our subjects, (ii) stimulus volumes were small $(0.1,0.3$, and $0.5 \mathrm{ml}$ as equivalent to 1,3 , and 5 drops) and may be closer to sensory thresholds, and (iii) continuous preexisting intrauterine swallowing reflexes to amniotic fluid ingestion (none of the subjects had antecedent evidence of polyhydramnios).

Similar to proximal aerodigestive reflexes, LESRR is evident in response to an esophageal bolus, and undergoes further maturation in pre-term infants (12). The current results demonstrate maturational changes in LESRR resulting from liquid pharyngeal stimulation (PLESRR) regarding magnitude and duration. Increased magnitude of PLESRR with maturation may facilitate esophageal clearance of a swallowed bolus or gastro-esophageal refluxate $(5,12,13)$. Decreased duration of PLESRR with maturation may help make the infant less vulnerable to GER because the lower sphincter returns to baseline tonicity more quickly. These changes in reflex functions likely characterize the maturation of vagal nerve function and central pattern generation activity in the brain stem. Similarities or changes in aerodigestive protective reflexes, such as PRS and PLESRR may represent maturational similarities or differences in the modulation of excitatory and inhibitory pathways during pharyngeal stimulation (Figure 4). Functional neural mapping of these phenomena has been made possible (14-17), and neural pathways naming the glossopharyngeal (cranial nerve IX) and vagus (cranial nerve $\mathrm{X}$ ) nerves as having control over UES and LES reflexes have been established $(5,18,19)$. As the presence of reflexes implies intact neural circuitry, results indicate skeletal muscle circuitry did not undergo significant longitudinal change during this time period, but smooth muscle circuitry, while intact during both times of study, may be modified. 


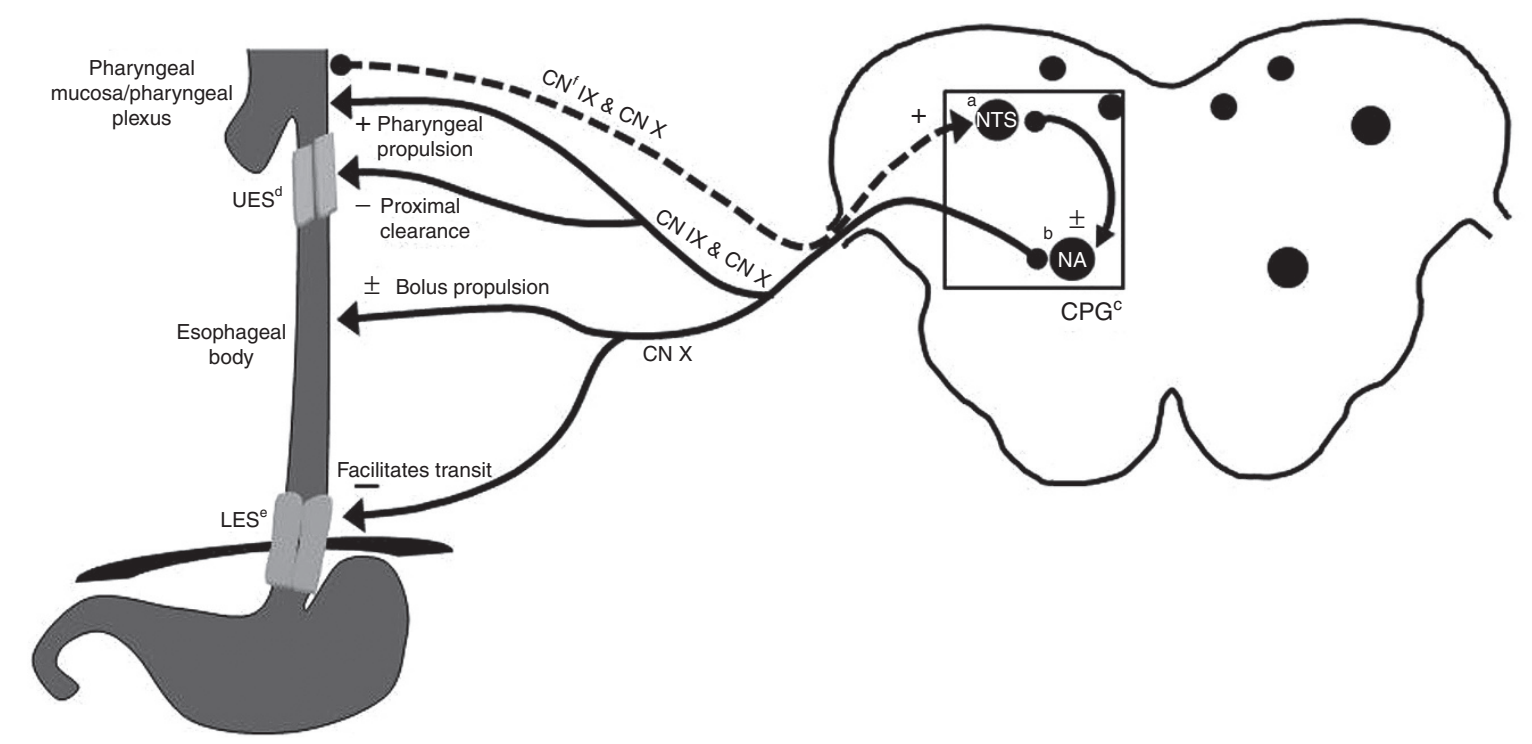

Figure 4 Neural circuits for PRS and PLESRR. ${ }^{a}$ Nucleus tractus solitarius. ${ }^{b}$ Nucleus ambiguus. ${ }^{\circ}$ Central pattern generator. ${ }^{\mathrm{d}}$ Upper esophageal sphincter. eLower esophageal sphincter. ${ }^{\mathrm{f}}$ Cranial nerve; dotted arrow: afferent pathway; dashed arrow: efferent pathway. Upon pharyngeal stimulation, afferents from the glossopharyngeal and vagus nerves signal efferent responses in the pharynx (PRS), upper esophageal sphincter, esophageal body, and lower esophageal sphincter (PLESRR). PLESRR, pharyngo-lower esophageal sphincter relaxation reflex; PRS, pharyngeal reflexive swallow.

The underlying mechanisms for liquid stimulations that resulted in safe esophageal transit included multiple PRS (volume dependent) (7), and prolonged LES relaxation that was also greater in magnitude, when compared with air. Because the water infusion volumes (given in triplicate as per our protocol) were similar at times- 1 and -2 , the differences in LES kinetics were attributed due to longitudinal maturation, and not due to the multiplicity of pharyngeal responses. However, similar observations with air infusion volumes (also given in triplicate as per our protocol) were not noted across longitudinal maturation. These findings support that liquid sensitive receptors advance with maturation, whereas mechanosensitive stimulation with air remains similar. Clinical relevance lies in the changes in aerodigestive transit from fetal to neonatal life. The aerodigestive tract in the fetus is only exposed to the liquid amniotic fluid stimulating the liquid sensitive receptors resulting in fetal swallowing; in contrast, postnatally, the aerodigestive tract has exposure to both, air and liquids. Air stimulus is rather chronic stimulus (with each breath), contrasting episodic liquid provocations such as many happen during salivary swallows or feeding swallows. Thus, adaptation of the pharyngeal receptor sensitivity may modify the motor responses at the pharyngeal, UES, esophageal or LES level. The above clinical findings indicate that inhibitory pathways to the lower esophageal sphincter are activated during pharyngeal provocation, and further develop and differentiate with maturation.

The physiological role of PRS is to clear the aerodigestive tract of any foreign substance, thus protecting against airway aspiration. Mechano-sensitive provocations with air puffs have resulted in a greater number of completely propagated esophageal peristalsis events along with abbreviated PLESRR. Abrupt swallowing of air with esophageal peristalsis may occur either in the presence or absence of feeds, as well as when the infant is crying, and is a possible causation for distended stomachs needing decompression, necessitating burping after feeding. The shorter duration of LES relaxation associated with air infusions may be protecting against gastro-esophageal reflux that may potentially occur from fundal stimulation and transient lower esophageal sphincter relaxation. The greater rate of completed propagation observed in response to air infusions (vs. liquid) may be due to the significantly fewer multiple PRS responses to air infusions, or air may clear more easily than liquids, requiring less effort. There is no difference in frequency recruitment or volumetric response to air stimuli during maturation. This may be due to hyposensitivity to air.

While the swallowing reflex is well developed in orally feeding healthy term infants, development of this reflex as infants adapt from prematurity or other perinatal problems, resulting in a prolonged NICU stay is unknown. Infants who have had a NICU stay display delayed feeding milestones, oral pooling, oromotor inertia, delayed initiation of swallowing, laryngeal penetration, airway aspiration, and cardiopulmonary events $(20,21)$. The sensory and motor aspects of pharyngeal reflexes favoring airway protection or facilitating peristalsis as the infant matures have been clarified by this study.

Aerodigestive protective mechanisms in the form of PRS and PLESRR have characteristics which both accelerate with maturation in preterm born infants and are similar during both times of study, as evidenced by the sensory-motor results. These changes in reflex functions characterize the maturation of the vagus nerve and central pattern generator activity in the brain stem. Changes in aerodigestive protective reflexes as well as inhibitory regulation of the lower esophageal sphincter, in response to media with maturation, may represent differences and advancement in the modulation of excitatory and inhibitory pathways. 
Limitations of this study include the lack of a term healthy infant comparator group. This is an unavoidable issue when conducting manometry studies in infants because there are feasibility and ethical recruitment obstacles. Data show a high degree of variability when plotted longitudinally, indicating that maturation is a process in continuum, and widening the gap between studies to effectively establish the time period associated with peak maturation may be of benefit in the future. However, the results from this longitudinal study contribute to knowledge of the development of both skeletal and smooth muscle responses to pharyngeal stimulation after premature birth, as the infant nears full oral feeding ability. Future directions include continued efforts to define what a "normal" response is, in addition to ways premature infant skills may developmentally deviate from or hold similarities to these sorts of "normal" responses.

\section{METHODS}

Protocol approval was sought from the Institutional Research Review Board at the Nationwide Children's Hospital Research Institute. The study protocol conforms to the guidelines of the IRB policy and the health insurance portability and accountability acts (HIPAA). Informed consents and HIPAA authorization were obtained from parents prior to study. Enrollment and studies were performed consecutively, based on inclusion and exclusion criteria, as well as scientific appropriateness, parental consenting, and ability to perform second studies to evaluate longitudinal maturation. Parents were not compensated for allowing infants to participate in the study. At time-1, all infants had a gavage tube and were exclusively gavage fed, whereas at time-2, the majority of infants had oral feeding skills

\section{Manometry Methods}

Multimodal pharyngeal provocations, using graded volumes of air and sterile water, were given and manometric recordings were made as previously described $(7-10,17)$. Briefly, the catheter assembly (Dentsleeve International, Mui Scientific, Mississauga, Ontario, Canada) was connected to the pneumohydraulic micromanometric water perfusion system via the resistors, pressure transducers (TNF-R disposable pressure transducers) and amplifiers (Solar modules, Solar 2, MMS medical instruments, Dover, NH). A 6 French manometry catheter assembly with dual sleeves (positioned at the UES and LES), 4 side-ports recording from the pharynx, the proximal-, middle-, and distal esophageal loci, in addition to a terminal gastric recording port was used. The micromanometric water perfusion rates were $0.02 \mathrm{ml} / \mathrm{min} /$ port for esophageal ports, $0.01 \mathrm{ml} / \mathrm{min} /$ port for the pharyngeal port, and $0.04 \mathrm{ml} / \mathrm{min} /$ port for the sleeves. The catheter was passed nasally while the infant lay supine and unsedated with the transducers at the level of the subject's esophagus. The manometry catheter was properly positioned using the pull through technique to identify high-pressure zones, and was well secured. Infants were allowed to adapt to catheter placement before beginning the experimental protocol.

\section{Manometric Experimental Protocol}

After about fifteen minutes of adaptation to catheter placement, upstream and downstream responses to graded volumes of pharyngeal infusions (graded stimuli, $0.1 \mathrm{ml}, 0.3 \mathrm{ml}, 0.5 \mathrm{ml}$ air and water) were evaluated. Infusions were given in increasing sequential order. Time lapse between stimuli administration was variable, and depended on the extent of the patient response to stimulus. Infusions were given upon restoration of aerodigestive quiescence. The rationale for giving infusions in this manner was to simulate a natural pharyngeal bolus that can have varying volumes. This is an attempt to evaluate the patient's ability to evoke appropriate pharyngeal reflexes based on mechanosensitive (air infusions) and osmosensitive (water infusions) stimulus. The specific responses of interest were pharyngeal reflexive swallow (PRS) and pharyngo-lower esophageal sphincter relaxation reflex (PLESRR). Respiratory waveform characteristics were monitored using Respiratory Inductance Plethysmography methods for subject safety.

\section{Manometry Data Analysis}

Characteristics for manometry data analysis have been described before $(7-11,17,19,22)$ and are briefly defined as follows: (i) Pharyngeal Reflexive Swallow (PRS) was defined as a deglutition response to pharyngeal stimulation for which onset is indicated by the presence of a pharyngeal waveform in association with UES relaxation, after which propagation into the proximal, middle, and distal esophageal segments is present, and is further accompanied by LES relaxation; (ii) Pharyngo-Lower Esophageal Sphincter Relaxation Reflex (PLESRR) was defined as LES relaxation of at least $5 \mathrm{mmHg}$ below LES resting pressure after stimulus onset. Response time from infusion onset to PLESRR was taken as the duration from the infusion onset to LES relaxation. Nadir duration is the time across the lowest LES pressure to the time it recovers to $5.0 \mathrm{mmHg}$. LES resting pressure was evaluated before the onset of the infusions relative to gastric pressure at end expiration. PLESRR magnitude was defined as the difference in pressure between resting LES pressure and maximum relaxation pressure.; (iii) Threshold volume was determined by the presence of $\mathrm{a}>50 \%$ response to an infusion and a $>50 \%$ response rate at subsequent increments; (iv) Multiple PRS was the presence of more than one PRS in response to a given infusion.

\section{Statistical Analysis}

Reflexes were visually identified at the time of study, and co-investigators were trained to recognize them during subsequent waveform analysis. Data were analyzed using SAS version 9.3 (SAS Institute, Cary, NC). GEE models were applied to establish the effect of infusion volume and the media effect on response type. Linear Mixed Models were used to establish the effect of media on magnitude and duration of PLESRR, response time from onset of infusion to onset of PRS or PLESRR, as well as establishing threshold volumes. In order to understand the overall responses to different physical characteristics of stimuli (air or water), responses to pooled air vs. water stimuli were evaluated and compared. Data are presented as mean $\pm \mathrm{SE}, \%$, or as otherwise stated. $P$ values $<0.05$ were considered significant.

\section{ACKNOWLEDGMENTS}

The authors are grateful to Juan Peng, MS, for statistical analysis; Vanessa Parks, BA, for technical support; and Rebecca Moore, RNC, for nursing coordination.

\section{STATEMENT OF FINANCIAL SUPPORT}

This study has been supported in part by National Institute of Health grant PO1 DK 068051 (S.R.J./R.S.) and NIH RO1 DK 068158 (S.R.J.).

Disclosure: The authors declare no conflict of interest.

\section{REFERENCES}

1. Burklow KA, McGrath AM, Allred KE, Rudolph CD. Parent perceptions of mealtime behaviors in children fed enterally. Nutr Clin Pract 2002;17:291-5.

2. Rommel N, De Meyer AM, Feenstra L, Veereman-Wauters G. The complexity of feeding problems in 700 infants and young children presenting to a tertiary care institution. J Pediatr Gastroenterol Nutr 2003;37:75-84.

3. Bingham PM. Deprivation and dysphagia in premature infants. J Child Neurol 2009;24:743-9.

4. Societal costs of preterm birth. In: Behrman RE, Butler AS, eds. Preterm Birth: Causes, Consequences, and Prevention. Washington, DC: The National Academic Press, 2007:398-424.

5. Jadcherla SR. Nascent Pharynx, physiology, reflexes. In: Shaker R, Postma G, Belafsky P, Easterling C, eds. Principles of Deglutition: A Multidisciplinary Text for Swallowing and its Disorders. New York: Springer Science, 2012:227-30.

6. Rommel N, van Wijk M, Boets B, et al. Development of pharyngoesophageal physiology during swallowing in the preterm infant. Neurogastroenterol Motil 2011;23:e401-8. 
7. Jadcherla SR, Gupta A, Stoner E, Fernandez S, Shaker R. Pharyngeal swallowing: defining pharyngeal and upper esophageal sphincter relationships in human neonates. J Pediatr 2007;151:597-603.

8. Jadcherla SR, Duong HQ, Hoffmann RG, Shaker R. Esophageal body and upper esophageal sphincter motor responses to esophageal provocation during maturation in preterm newborns. J Pediatr 2003;143: 31-8.

9. Jadcherla SR, Duong HQ, Hofmann C, Hoffmann R, Shaker R. Characteristics of upper oesophageal sphincter and oesophageal body during maturation in healthy human neonates compared with adults. Neurogastroenterol Motil 2005; 17:663-70.

10. Jadcherla SR, Hoffmann RG, Shaker R. Effect of maturation of the magnitude of mechanosensitive and chemosensitive reflexes in the premature human esophagus. J Pediatr 2006;149:77-82.

11. Gupta A, Gulati P, Kim W, Fernandez S, Shaker R, Jadcherla SR. Effect of postnatal maturation on the mechanisms of esophageal propulsion in preterm human neonates: primary and secondary peristalsis. Am J Gastroenterol 2009;104:411-9.

12. Shi G, Ergun GA, Manka M, Kahrilas PJ. Lower esophageal sphincter relaxation characteristics using a sleeve sensor in clinical manometry. Am J Gastroenterol 1998;93:2373-9.

13. Mittal RK, Balaban DH. The esophagogastric junction. N Engl J Med 1997;336:924-32.

14. Martin RE, Sessle BJ. The role of the cerebral cortex in swallowing. Dysphagia 1993;8:195-202.
15. Mosier K, Patel R, Liu WC, Kalnin A, Maldjian J, Baredes S. Cortical representation of swallowing in normal adults: functional implications. Laryngoscope 1999;109:1417-23.

16. Jadcherla SR, Gupta A, Coley BD, Fernandez S, Shaker R. Esophago-glottal closure reflex in human infants: a novel reflex elicited with concurrent manometry and ultrasonography. Am J Gastroenterol 2007;102:2286-93.

17. Jadcherla SR, Gupta A, Wang M, Coley BD, Fernandez S, Shaker R. Definition and implications of novel pharyngo-glottal reflex in human infants using concurrent manometry ultrasonography. Am J Gastroenterol 2009;104:2572-82.

18. Jadcherla SR, Shaker R. Physiology of aerodigestive reflexes in neonates and adults. In: Johnson L, ed. Physiology of the Gastrointestinal Tract. 5th edn. Oxford: Academic Press, 2012:893-918.

19. Pena EM, Parks VN, Peng J, et al. Lower esophageal sphincter relaxation reflex kinetics: effects of peristaltic reflexes and maturation in human premature neonates. Am J Physiol Gastrointest Liver Physiol 2010;299:G1386-95.

20. Lefton-Greif MA, Arvedson JC. Pediatric feeding and swallowing disorders: state of health, population trends, and application of the international classification of functioning, disability, and health. Semin Speech Lang 2007;28:161-5.

21. Arvedson JC. Assessment of pediatric dysphagia and feeding disorders: clinical and instrumental approaches. Dev Disabil Res Rev 2008;14:118-27.

22. Jadcherla SR, Chan CY, Fernandez S, Splaingard M. Maturation of upstream and downstream esophageal reflexes in human premature neonates: the role of sleep and awake states. Am J Physiol Gastrointest Liver Physiol 2013;305:G649-58. 